\title{
CAPACIDAD DE CARGA ASIGNABLE AL AGROECOTURISMO EN ÁREAS PROTEGIDAS DE BOLIVIA
}

\author{
Mariana Meave Acuña ${ }^{1}$ \\ Diosey Ramón Lugo-Morin² \\ Recibido el 25 de septiembre de 2014, aprobado el 5 de mayo de 2015 y \\ actualizado el octubre 28 de 2015
}

\section{DOI: 10.17151/luaz.2016.42.8}

\section{RESUMEN}

Este documento tiene como objetivo valorar la capacidad de carga asignable al agroturismo en áreas protegidas de Bolivia en el marco de la economía ecológica. En esta nación, el agroturismo puede constituirse en una práctica novedosa para hacer que los visitantes de áreas protegidas sean partícipes de las actividades de desarrollo económico local. Además, permite determinar la cantidad de personas que pueden visitar un sitio protegido sin alterar el equilibrio de los ecosistemas que alberga. Metodológicamente se empleó un enfoque mixto (cuantitativo y cualitativo) apoyado con la medición tradicional de carga turística física, real y efectiva.

En el caso de los agroecosistemas de las áreas protegidas de Bolivia se puede observar que existe capacidad de carga suficiente para su operación que actualmente que alcanza el $96 \%$. También, es posible mejorarla e incrementarla en función de las características ecológicas, físicas y sociales de estos sitios. Por otra parte es importante resaltar la importancia de los ingresos económicos obtenidos diarios de un área protegida individual que corresponde a 404 Bs. en promedio. Finalmente, el empleo de la economía ecológica permite establecer el valor y aprovechamiento de los recursos existentes en las áreas protegidas de interés nacional de Bolivia. De esta manera, es posible mejorar la gestión de sitios protegidas a nivel ambiental y financiero.

\section{PALABRAS CLAVE}

Agroecoturismo, capacidad de carga turística, visitantes, gestión, áreas protegidas.

\section{ALLOCABLE CAPACITY TO AGROECOTOURISM IN BOLIVIAN PROTECTED AREAS}

\section{ABSTRACT}

This document has the objective to value the allocable capacity assigned to agrotourism in Bolivian protected areas within the framework of ecological economy, Agroecotourism in this nation can become an innovating alternative to enhance the participation of protected areas visitors in local economic activities development. Furthermore, it allows quantifying the amount of people who can visit a protected site without disturbing the ecosystems equilibrium in the area. Methodologically, a mixed approach (quantitative and qualitative) was applied based on the traditional measuring of physical, real and effective tourism allocable capacity. In the case of the Bolivian agroecosystems protected areas it is possible to observe that there is enough allocable capacity for its operation, which is currently reaching $96 \%$. In addition, it is possible to improve it and increase it according to the ecological, physical and social characteristics of these sites. On 
the other hand, it is possible to highlight the importance of the economical revenues obtained daily in an individual protected area that corresponds to an average of 404 Bs. Finally, the application of ecological economy allows establishing the value and use of existing resources in the protected areas of national interest in Bolivia. In this way, it is possible to improve protected sites management in financial and environmental terms.

KEY WORDS: Agroecotourism, tourism allocable capacity, visitors, management, protected areas.

\section{INTRODUCCIÓN}

El turismo es una herramienta para el desarrollo de la economía de pueblos indígenas y comunidades campesinas. El agroecoturismo permite la participación plena y efectiva de los habitantes indígenas y locales en la gestión de áreas protegidas. También, brinda oportunidades de generación de ingresos (Von Lossau y Li, 2011). En las circunstancias adecuadas, ha demostrado ser uno de los medios más eficaces para financiar la preservación de la biodiversidad. El paisaje rural, por lo general, es una combinación de ecosistemas agrícolas y silvestres, que se encuentran entre los recursos más importantes para el desarrollo turístico (SERNAP, 2011). En este sentido, se pretende propiciar la generación de recursos económicos sin afectar a esos ecosistemas.

El turismo sostenible se define como la actividad que atiende a las necesidades de los visitantes actuales y de las regiones receptoras; protegiendo al mismo tiempo oportunidades futuras. Se centra en la gestión de los recursos disponibles a fin de satisfacer los requerimientos económicos, sociales y ambientales en el marco del respeto de la integridad cultural, los procesos ecológicos esenciales, la diversidad biológica y los ecosistemas existentes (CESD, 2006).

El ecoturismo es aquella modalidad turística recreativa ambientalmente responsable de visita de áreas naturales, evitando disturbar los ecosistemas existentes. Tiene el fin de apreciar y estudiar los atractivos naturales (paisaje, flora y fauna silvestre) de dichas zonas, así como toda manifestación cultural presente o pasada del lugar, a través de un proceso que promueve la preservación ambiental y propicia el involucramiento activo del visitante y el bienestar de la población local (Pedersen, 2005).

El agroturismo corresponde a la simbiosis turismo-agricultura. Es un segmento de mercado clave para el turismo, ambiental y socialmente responsable en áreas rurales. Tiene la particularidad de ubicarse en granjas, preferentemente activas. Los emplazamientos dedicados a esta actividad se encuentran en los alrededores de tierras agropecuarias, pudiendo hacer partícipe al visitante de sus operaciones (El-Hage y Williamson, 2004). También, se refiere a las nociones y principios ecológicos en el estudio, diseño y control de sistemas agrícolas (Camacho y Ariosa, 2000). En este caso se aplicará el enfoque tradicional de medición de carga turística física, real y efectiva desarrollado para sitios protegidos.

El agroecoturismo permite armonizar el paisaje rural silvestre y sus agroecosistemas transformándose en un recurso clave para el desarrollo, permitiendo la preservación de la biodiversidad y del patrimonio histórico cultural (El-Hage y Williamson, 2004). El turismo ecoorgánico corresponde a la evolución del agroecoturismo en granjas orgánicas que permite valorizar aspectos específicos que ofrecen los sitios de cultivo, lo que proporciona valor agregado a las actividades de protección ambiental (El-Hage y Williamson, 2004). 
Los agroecosistemas de las áreas protegidas de Bolivia tienen una capacidad de carga turística suficiente para su operación que actualmente que alcanza un $96 \%$. También, es posible mejorar e incrementar esta capacidad a través de las características ecológicas, físicas y sociales de estos sitios. Por otra parte es importante resaltar la importancia de los ingresos económicos generados diariamente a nivel nacional que corresponde a 8.885, 66 [Bs./día].

El resultado final del estudio permite realizar la monetarización de las actividades agroecoturísticas llevadas a cabo en áreas protegidas de interés nacional de Bolivia. De esta forma, es posible garantizar la sustentabilidad de la gestión de estos sitios protegidos.

\section{Impactos del turismo en áreas protegidas}

A menudo, la magnitud del uso en zonas turísticas no es proporcional al impacto ambiental. Es decir que el uso inicial, a pesar de ser moderado, puede generar la mayor parte del daño, en tanto que el uso adicional tiene efectos progresivamente menores. En el caso de recursos como los suelos y la vegetación, la mayor parte del daño tiende a ocurrir en bajos niveles de utilización.

La resistencia y la resiliencia son los dos principales factores del impacto. La resistencia es la capacidad de absorber el uso sin sufrir una perturbación; la resiliencia es la capacidad de retornar al estado inicial después de haber sufrido una modificación (Pedersen, 2005). En áreas protegidas, la resistencia es una medida de la capacidad de distintos ambientes y culturas de asimilar el cambio. Los pueblos indígenas y comunidades campesinas han sabido preservar su cultura durante varias generaciones pese a la presencia de turistas. En los sitios naturales, los suelos fértiles son más aptos para resistir el uso y mantener su vegetación; los suelos poco profundos tienen pocas probabilidades de soportar una actividad intensiva.

\section{Capacidad de carga turística de áreas protegidas}

La capacidad de carga es un método comprensible que permite definir el número máximo de visitas que puede recibir un área protegida en función a sus características físicas, biológicas y de manejo. También está asociada al nivel de uso para la satisfacción de los beneficiarios y los impactos en las estructuras económicas, sociales y culturales del entorno (Cifuentes et al., 1999; Puente, Pérez y Solís, 2011). Este indicador puede variar en función de la localización del sitio, la estacionalidad, el comportamiento del usuario, el diseño de las instalaciones existentes, el estado de preservación de los recursos del área natural, el acceso a servicios básicos, etc. (Báez y Acuña, 2003).

El término de capacidad de carga turística se refiere al número de personas cuya presencia se puede permitir en un área determinada, sin riesgo de que el sitio y la experiencia de los visitantes resulten perjudicados. En tanto que la capacidad de carga ecológica, se refiere al límite hasta el cual un ecosistema puede tolerar la interferencia humana manteniendo un funcionamiento sostenible (Pedersen, 2005).

Determinar una cifra precisa de capacidad de carga turística puede dar la falsa impresión de que un área protegida está salvaguardada cuando en realidad no es así. Aunque exista un límite fijo, eso no evitará que algunos visitantes perturben los ecosistemas existentes (Romero, 2014). Es necesario que los modelos actuales de gestión de visitantes permitan la formulación de indicadores de supervisión. 
Existen tres características que influyen en la manera de aplicación de la metodología de medición de la capacidad de carga asociadas al lugar, al tipo de turismo y a la interface ambiental presente (Coccossis et al., 2001), las cuales se describen como sigue.

i) Particularidades de las ecorregiones estudiadas. Provee la estructura básica para llevar a cabo las actividades turísticas. En términos de recursos naturales, vulnerabilidad de los ecosistemas locales, la densidad poblacional, la estructura económica y cultural. Hasta cierto límite, estos rasgos determinan su resiliencia a las presiones del turismo.

ii) Tipo de turismo. Se refiere al comportamiento de los visitantes, el estado de las comunidades locales, la calidad ambiental del sitio. El tipo de turismo puede ser definido en función a las razones de la visita, el modo de transporte, la frecuencia de las visitas, las actividades efectuadas, etc. En este caso, las diferencias entre turistas están dadas por sus expectativas, actitudes y el modo de proceder de cada uno de ellos.

iii) Interface del visitante y los ecosistemas. Tiene un carácter dual porque está influenciada por los dos factores anteriores. Se centra en el aspecto espacial, el ciclo de vida del sitio de destino, el nivel de organización y el régimen de gestión del mismo. Se expresa en términos de impacto ambiental y de degradación del entorno.

Existen diversos índices estándar de capacidad de carga general en sitios turísticos y recreativos. Los que deben adecuarse al tipo de actividad llevada a cabo, la densidad de uso y el ambiente natural. Por esta razón, se define como coeficiente de capacidad de carga permisible para el agroecoturismo en áreas protegidas un valor de $45\left[\mathrm{~km}^{2} /\right.$ visitante], debido a que se trata de un uso destinado a la preservación (Botero et al., 2008).

El dimensionamiento de la capacidad de carga turística en el territorio correspondiente a áreas protegidas, se desarrolla en los tres siguientes niveles (Tudela y Giménez, 2008):

a) Capacidad de Carga Física (CCF). Es la cantidad máxima de visitas que se pueden hacer al sitio durante un día.

b) Capacidad de Carga Real (CCR). Es el límite máximo de visitas asignables a un sitio tras aplicar factores de corrección en función de sus características particulares.

c) Capacidad de Carga Efectiva (CCE). Es el número máximo de visitantes al día que se puede permitir para ordenarlas y manejarlas de manera adecuada. Se obtiene comparando la capacidad de carga real (CCR) y la capacidad de manejo (CM) por parte de la administración del sitio. La capacidad de manejo (CM) se define como la suma de variables de funcionamiento como la infraestructura, el personal y el equipamiento, cuyos valores cualitativos pueden ser ponderados con base en la escala porcentual establecida en la norma ISO 10004. Los valores asignados para la evaluación de criterios se presentan en la Tabla 1. 
Tabla 1. Escala porcentual de evaluación de criterios de funcionamiento

\begin{tabular}{|c|c|c|}
\hline Valor & Porcentaje & Calificación \\
\hline 0 & $\leq 35$ & Insatisfactorio \\
\hline 1 & $36-50$ & Poco satisfactorio \\
\hline 2 & $51-75$ & Medianamente satisfactorio \\
\hline 3 & $76-89$ & Satisfactorio \\
\hline 4 & $\geq 90$ & Muy satisfactorio \\
\hline
\end{tabular}

Fuente: Cifuentes et al. (1999).

Los tres niveles de capacidad de carga tienen la sucesión que se presenta en la Ecuación 1.

Ecuación 1. Orden de las capacidades de carga

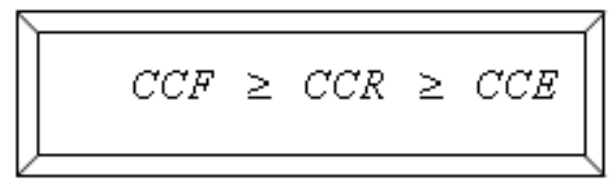

Fuente: Cifuentes et al. (1999).

La secuencia metodológica aplicada para el dimensionamiento de la capacidad de carga turística de áreas protegidas se muestra en la Figura 1.

Figura 1. Disposición de la metodología de la capacidad de carga

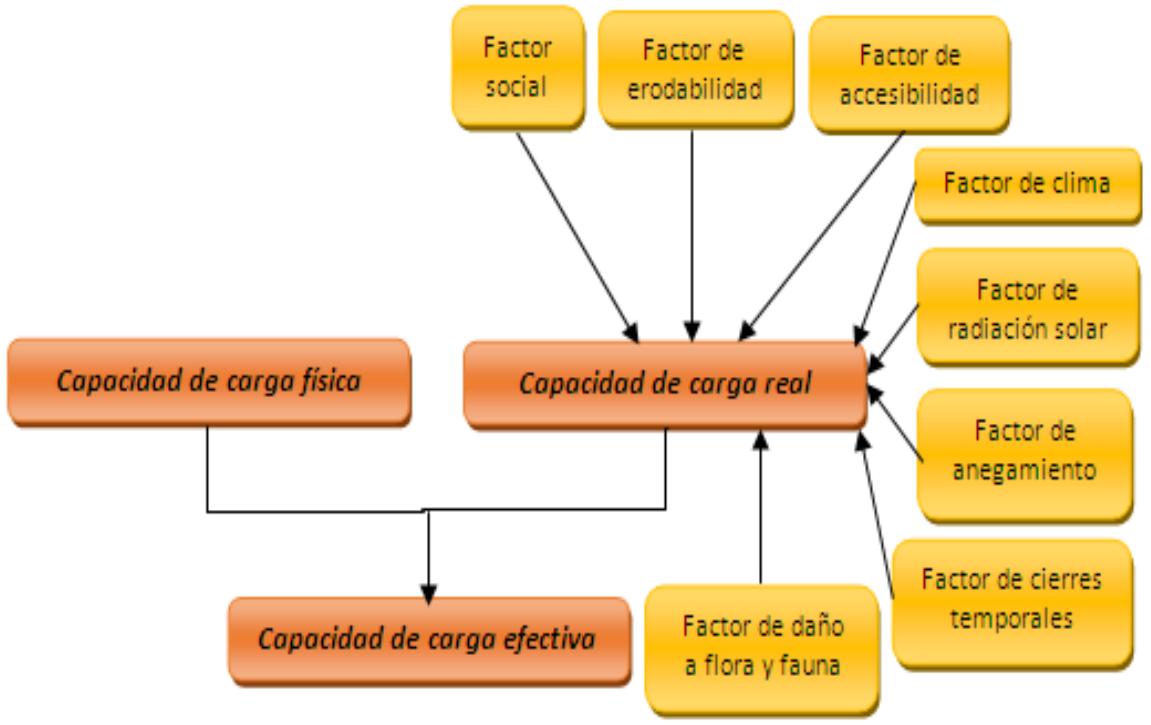

Fuente: adaptada de Tudela y Giménez (2008). 
Los coeficientes de corrección aplicables al caso de sitios protegidos en calidad de áreas recreativas son los siguientes: i) Factor social (FCsoc), el cual se encarga de limitar la afluencia de turistas en el aspecto de la superficie asignada, actividades realizadas, sociabilidad y nivel de ruido; ii) Factor de erodabilidad (FCer), respecto al pisoteo, compactación, pendiente y la granulometría del suelo; iii) Factor de accesibilidad (FCac), sobre el estado físico del terreno, el transporte, los servicios básicos existentes, los desembolsos que debe realizar el turista; iv) Factor climático (FCCl), el cual limita las visitas de acuerdo a las condiciones meteorológicas; v) Factor de radiación solar (FCrs), de acuerdo a la intensidad del sol; vi) Factor de anegamiento (FCan), en función de la estacionalidad de las precipitaciones pluviales; vii) Factor de cierres temporales (FCct), de acuerdo al mantenimiento de las instalaciones y viii) Factor de daño a la flora y fauna (FCdf), en función de la pérdida de biodiversidad.

\section{Gestión de impactos asociados a la visita de áreas protegidas}

Los indicadores de gestión son de vital importancia para la evaluación y mitigación de impactos ambientales que puedan ser generados por los visitantes de áreas protegidas (Amador et al., 1996). Los indicadores a tener en cuenta son de tipo ecológico, físico y social. Entre los aspectos a ser supervisados se pueden citar: abrasión de monumentos, estado de la vegetación, tamaño de los grupos, polución debida a la presencia humana, quejas de los turistas acerca del estado del sitio, erosión, perturbación de la fauna y la flora silvestres, quejas de miembros de la comunidad acerca de la degradación de los valores culturales, etc.

Una vez que se han establecido los indicadores y sus respectivos límites permisibles, un inventario determinará en qué medida los resultados existentes difieren de los deseados. Se trata de un proceso permanente en el que se hace el seguimiento de los indicadores de modo regular, a fin de detectar las tendencias y los cambios en los sitios estudiados.

\section{Metodología de medición de la capacidad de carga turística de áreas protegidas}

El procedimiento correspondiente al dimensionamiento de la capacidad de carga destinada a actividades de agroecoturismo en áreas protegidas tiene las siguientes cuatro fases principales (Cifuentes, 1992):

1) Análisis de la situación actual de actividades turísticas y gestión de áreas protegidas.

2) Descripción de los sitios de visita.

3) Presentación de factores que influyen en los sitios turísticos.

4) Determinación de la capacidad de carga para la zona seleccionada.

5) Valoración monetaria de las actividades turísticas.

Como se mencionó anteriormente, existen distintos coeficientes de corrección que son empleados a fin de obtener los valores de capacidad de carga, algunos de los cuales fueron especialmente desarrollados para áreas protegidas. En este sentido, es posible introducir la innovación de la descripción en detalle de los factores asociados a la calidad de la experiencia recreativa de los visitantes (Aranguren et al., 2008).

Por otra parte, es posible realizar la monetarización del beneficio de las visitas de áreas protegidas como bien ambiental, cuya preservación implica costos financieros a cargo de las autoridades y costos de oportunidad como los correspondientes a las actividades turísticas. El método tradicional podría enriquecerse al incluir los beneficios económicos hipotéticos que pueden 
obtenerse por la visita de estos sitios naturales. Esto permite estimar la disposición a pagar de los visitantes de las áreas naturales protegidas, por los beneficios sociales y ambientales que proporcionan (Martínez, Roca y Sánchez, 1998).

\section{Situación actual del turismo en Bolivia}

Es importante resaltar el avance en la actividad turística por parte de los pobladores de áreas protegidas. El emprendimiento del Chalalán, que corresponde al Parque Nacional y Área Natural de Manejo Integrado (PN y ANMI) Madidi, es una clara muestra de desarrollo de la comunidad Tacana-Quechua de esta ecorregión. Esta iniciativa genera alrededor de 1500 empleos para la población local. Una parte de los ingresos generados es reinvertida en centros de salud, infraestructura, educación, etc. Este modelo de turismo es ideal para promover la sostenibilidad (Verner et al., 2010).

Dentro del Sistema Nacional de Áreas Protegidas (SNAP) existen 34 emprendimientos en 11 áreas protegidas o sus respectivas zonas de amortiguación (ZA). Se han realizado bastantes esfuerzos por parte de entidades públicas y de organizaciones de la sociedad civil a fin de mejorar los servicios básicos existentes en estas regiones. Se puede señalar que durante la gestión 2010, la Reserva Nacional de Fauna Andina (RNFA) Eduardo Avaroa y el Parque Nacional y Área Natural de Manejo Integrado (PN y ANMI) Madidi, captaron el $82 \%$ del total de visitantes en áreas protegidas nacionales generando una recaudación de alrededor de 2'585.200 [Bs.]. Seguidas de los sitios protegidos Amboró, Cotapata, Carrasco y Sajama que recibieron el $16 \%$ de los turistas. Finalmente, el Parque Nacional (PN) Torotoro y el resto de las áreas protegidas nacionales representaron aproximadamente el $2 \%$ de la afluencia de visitantes (SERNAP, 2011).

\section{Descripción del área de estudio}

Bolivia posee 22 áreas protegidas (APs) de interés nacional, las que tienen una extensión de $1^{\prime} 081.927,54 \mathrm{~km}^{2}$, de las que $76.741,14 \mathrm{~km}^{2}$ corresponden a tierras comunales en las que se pueden llevar a cabo actividades agroecoturísticas, cuya distribución se presenta en la Figura 2. 
Figura 2. Distribución de tierras comunales en áress protegidss

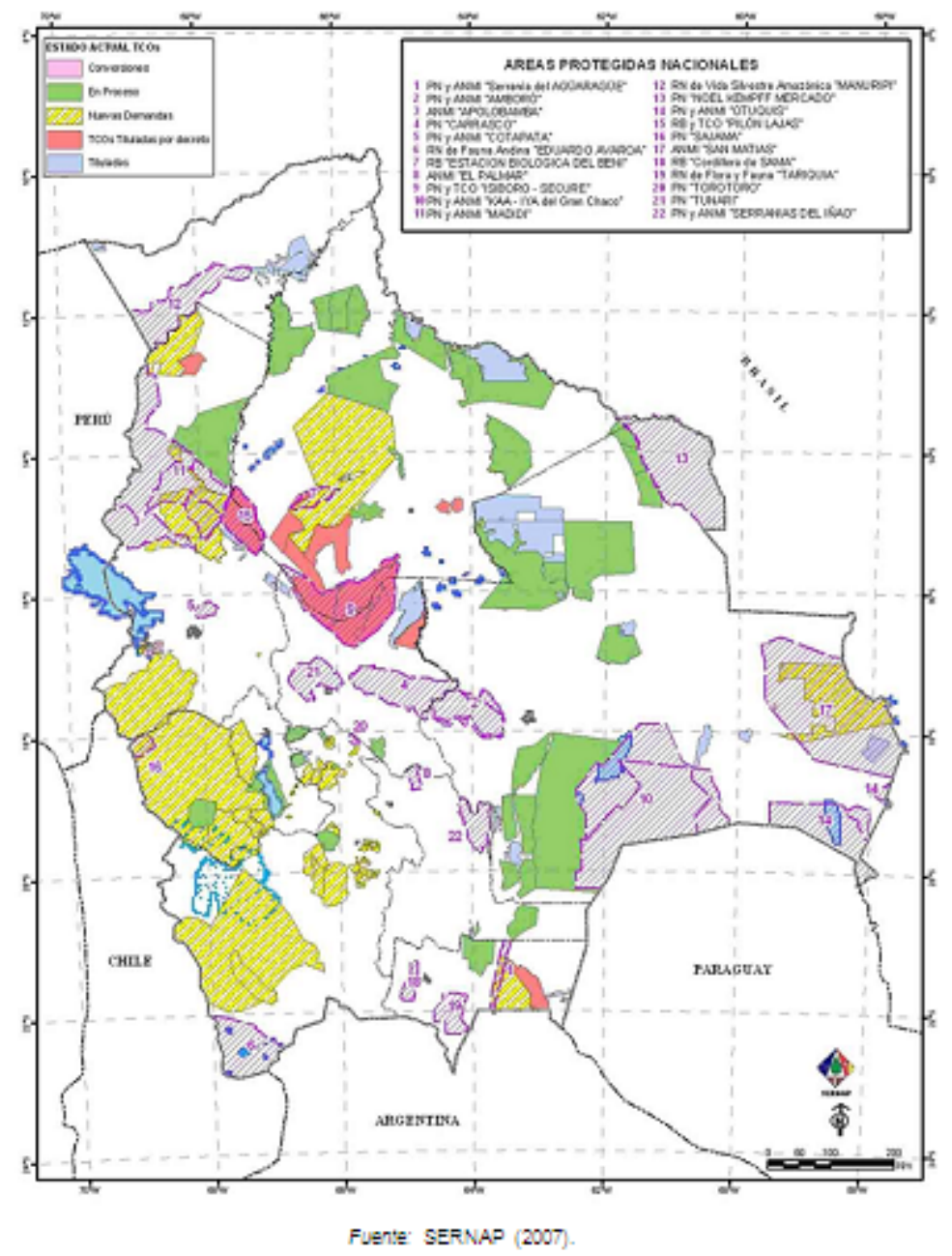

Haga clic para ampliar la images

Cabe mencionar que en los sitios protegidos viven alrededor de 200.000 habitantes. Además, aproximadamente el $70 \%$ de la población de estas zonas es de origen indígena.

Se pueden identificar 44 tierras comunitarias de origen (TCO), entre tituladas y demandadas, de acuerdo a su gestión: 5 están superpuestas totalmente [2 de doble condición (AP y TCO) y 3 dentro de áreas protegidas], 9 parcialmente y 30 en la zona de amortiguación (SERNAP, 2007). La importancia de estas zonas para sus habitantes está asociada a la protección de los derechos sobre la tierra, el uso de los recursos naturales, el mantenimiento de cuencas, la preservación de la biodiversidad y la valorización de su patrimonio cultural.

Asimismo, se tiene en cuenta que el número de guardaparques de los sitios protegidos de importancia nacional en Bolivia es de alrededor de 300 en la actualidad. A pesar de que existió un crecimiento numérico del cuerpo de protección, la proporción entre personal de vigilancia y superficie bajo resguardo resulta mínima. Es equivalente a un guardaparque por $600 \mathrm{~km}^{2}$ en promedio (SERNAP, 2007).

También, es necesario tener en cuenta que esta situación es aún más desfavorable en áreas protegidas de gran extensión y de difícil acceso como el 
Territorio Indígena y Parque Nacional Isiboro Securé (TIPNIS), Otuquis y San Matías en los que la zona cubierta es de entre 700 y $3.600\left[\mathrm{~km}^{2} /\right.$ guardaparque].

\section{Presentación de factores que influyen en las áreas protegidas}

Los factores que influyen en las áreas protegidas, la descripción de los mismos y sus respectivos coeficientes de corrección se presentan de manera detallada en la Tabla 2.

Tabla 2. Factores y coeficientes de corrección para áreas protegidas

\begin{tabular}{|c|c|c|}
\hline Factores & Descripción & Coeficientes \\
\hline \multirow{4}{*}{ Social } & Superficie & \multirow{4}{*}{0,84} \\
\hline & Actividades & \\
\hline & Sociabilidad & \\
\hline & Nivel de ruido & \\
\hline \multirow{3}{*}{ Erodabilidad } & Pisoteo y compactación & \multirow{3}{*}{0,54} \\
\hline & Pendiente & \\
\hline & Granulometría del suelo & \\
\hline \multirow{4}{*}{ Acceso } & Físico & \multirow{4}{*}{0,73} \\
\hline & Transporte & \\
\hline & Servicios & \\
\hline & Desembolsos & \\
\hline \multirow{2}{*}{ Clima } & Periodo lluvioso & \multirow{2}{*}{0,88} \\
\hline & Periodo seco & \\
\hline Radiación solar & Brillo & 0,85 \\
\hline Anegamiento & Desborde de cursos de agua & 0,80 \\
\hline Cierres temporales & Mantenimiento de instalaciones & 0,99 \\
\hline Daño a la flora y fauna & $\begin{array}{l}\text { Pérdida de biodiversidad (daño } \\
\text { a flora y fauna) }\end{array}$ & 1,00 \\
\hline
\end{tabular}

Fuente: elaboración propia con base en Cifuentes et al. (1999) y Aranguren et al. (2008).

\section{RESULTADOS OBTENIDOS}

En este acápite se lleva a cabo el procesamiento de los datos obtenidos, que fueron extraídos de la literatura sobre el agroecoturismo en áreas protegidas de interés nacional.

\section{Determinación de la capacidad de carga asignable al agroecoturismo}

Finalmente, fue posible aplicar la metodología de medición de capacidad de carga al agroecoturismo en las 22 áreas protegidas de interés nacional de Bolivia, como 
se muestra en la Figura 3. También, se pudieron estimar los ingresos generados por los visitantes de estos sitios protegidos.

Figura 3. Capacidad de carga asignable al agroecoturismo en áreas protegidas de Bolivia

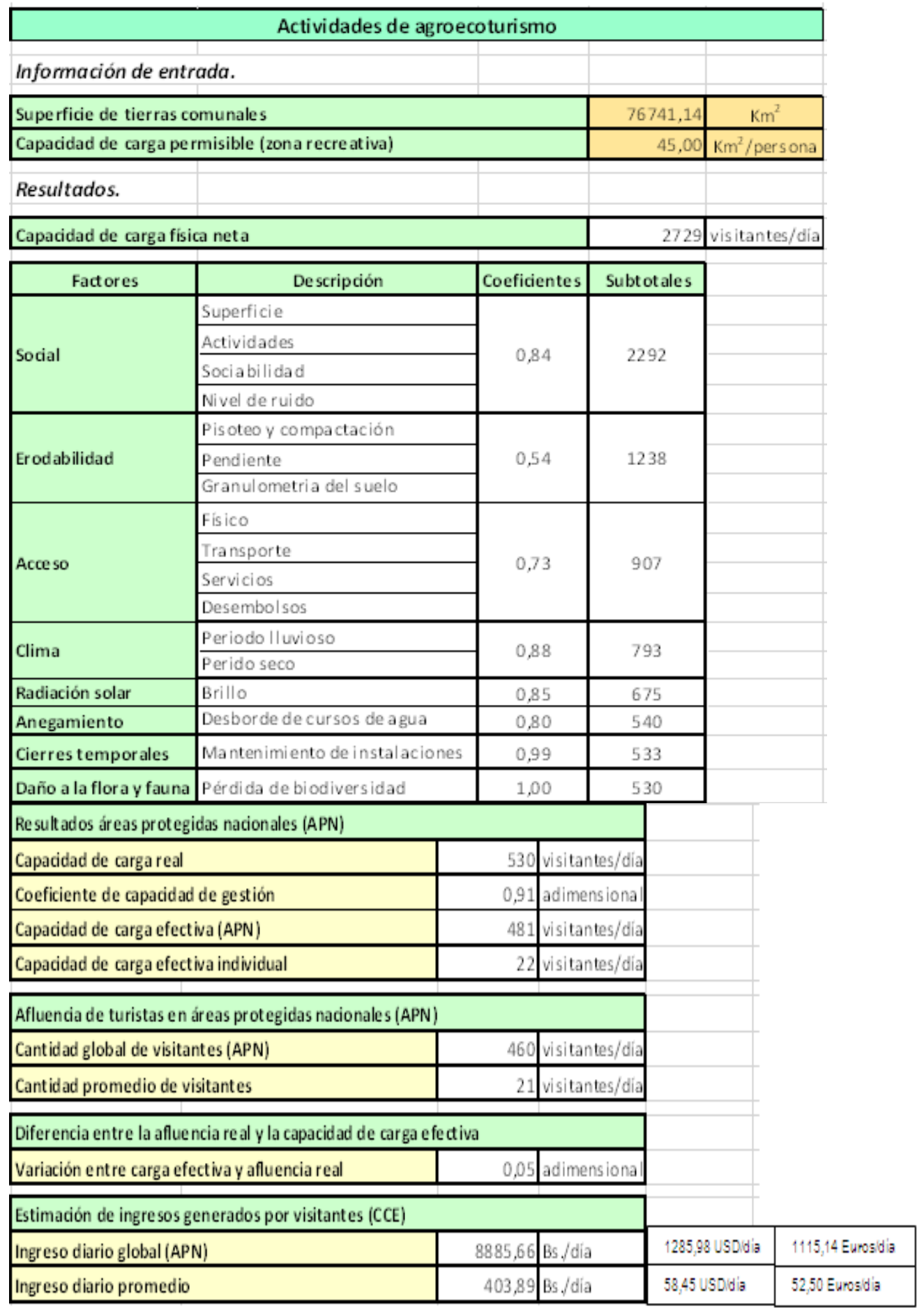

Fuente: adaptada de Cifuentes et al. (1999) y Aranguren et al. (2008). 
Se puede apreciar que la capacidad de carga física de actividades agroecoturísticas llevadas a cabo en áreas protegidas nacionales es de 2.729 [visitantes/día], la capacidad de carga real es de 530 [visitantes/día] y la capacidad de carga efectiva es de 481 [visitantes/día]. No obstante, la afluencia real de turistas es de 460 [visitantes/día]. Por tanto, se opera en un nivel inferior la capacidad de carga efectiva en un 4,39\%.

A su vez, se puede observar que se genera un ingreso diario por visitas de 8.885, 66 [Bs./día] en la totalidad de las áreas protegidas de interés nacional de Bolivia. El detalle de la valoración monetaria de las visitas en áreas protegidas se presenta en la Tabla 3.

Tabla 3. Ingresos generados por visitas de áreas protegidas nacionales

\begin{tabular}{|l|c|c|}
\hline \multicolumn{1}{|c|}{ Descripción } & Valor & Unidad \\
\hline Ingreso anual global (APs) visitadas frecuentemente & $2585.200,00$ & Bs./año \\
\hline Cantidad anual de visitantes & $135.244,00$ & visitantes/año \\
\hline Ingreso anual por visitante (APs) visitadas frecuentemente & 20,26 & Bs./visitante \\
\hline Ingreso anual global (APs) visitadas poco frecuentemente & $47.3091,60$ & Bs./año \\
\hline Cantidad anual de visitantes & $30.227,00$ & visitantes/año \\
\hline Ingreso anual por visitante (APs) visitadas poco frecuentemente & 17,38 & Bs./visitante \\
\hline Ingreso anual promedio total por visitante (APs) & 18,43 & Bs./visitante \\
\hline
\end{tabular}

Fuente: elaboración propia con base en Martínez, Roca y Sánchez (1998).

En este caso, se desglosan los beneficios generados por las áreas protegidas de interés nacional visitadas frecuentemente y aquellas que reciben pocos visitantes. Finalmente, se obtiene el ingreso anual promedio de ambas que es de 18,43 [Bs./visitante].

La representación gráfica de los beneficios percibidos por la visita de sitios protegidos nacionales se muestra en la Figura 4. La pendiente de los ingresos generados por espacios protegidos visitados masivamente y minoritariamente es ascendente. El área bajo ambas líneas mide en términos económicos la totalidad del beneficio obtenido por las actividades turísticas llevadas a cabo en estos sitios protegidos. Además, es posible deducir que algunos de ellos se ubican en lugares de difícil acceso, lo que justifica el hecho de que tengan muy pocos visitantes. A la inversa, el valor económico crece de manera proporcional respecto al número de visitas. No obstante, demasiados turistas pueden incidir negativamente en la preservación del entorno natural. 
Figura 4. Beneficios económicos percibidos por la visita de sitios protegidos

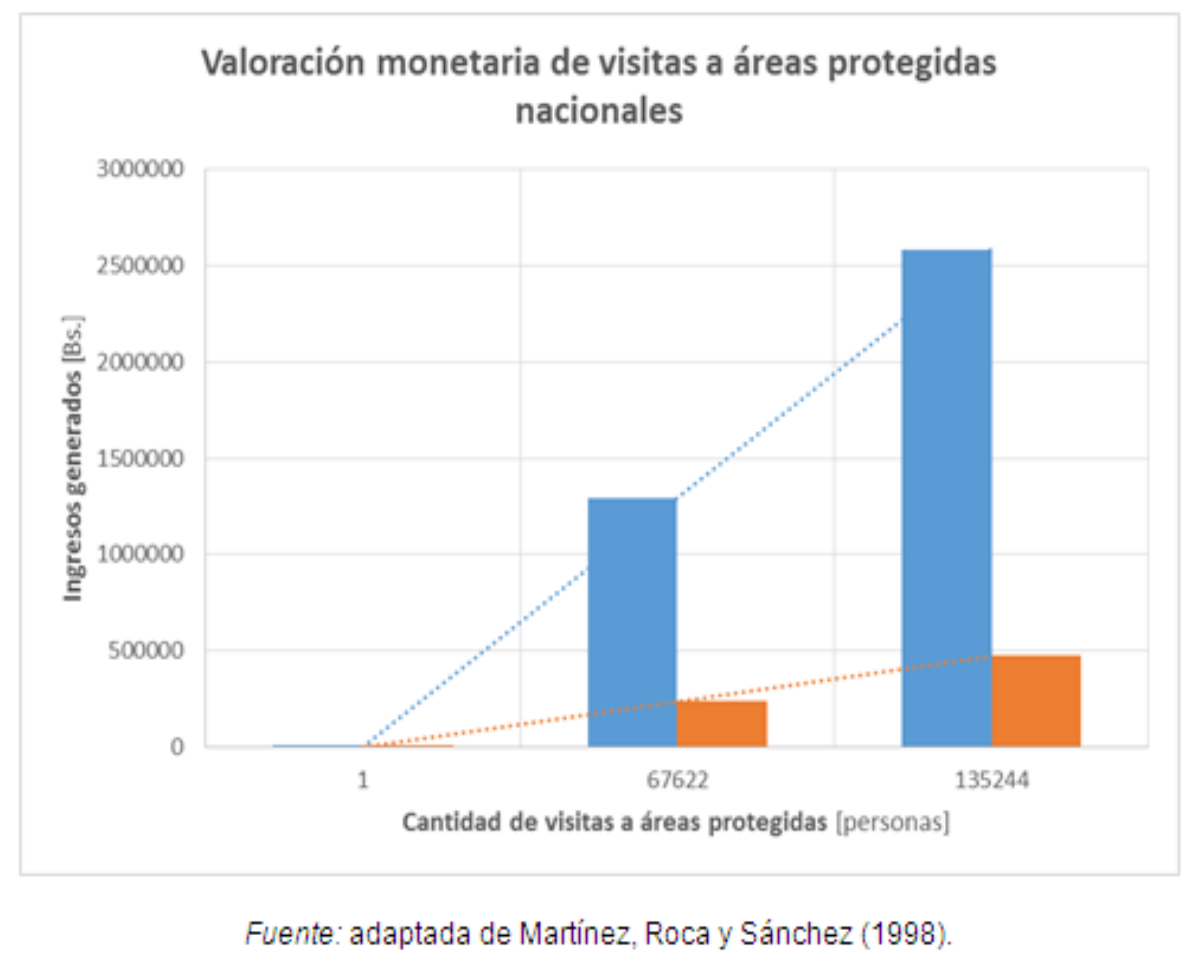

Estos beneficios pueden incrementarse en función de las mejoras de gestión que se apliquen respecto a la capacidad de carga turística de las áreas protegidas nacionales.

\section{Mecanismos de gestión de la carga turística de áreas protegidas}

La metodología de estimación del número permisible de visitantes en áreas protegidas es flexible y está sujeta a las variaciones existentes en el entorno y su gestión. Es importante, detectar los impactos de los visitantes a través de programas de supervisión y así evitar que se supere su límite. Los datos sobre el número de visitantes y sus preferencias, constituyen la mejor justificación de medidas que restrinjan la admisión. Las encuestas de visitantes podrían demostrar los efectos negativos de los fenómenos de aglomeración y el hacinamiento. En la Tabla 4 se presentan los indicadores, normas y métodos de evaluación de la experiencia de los visitantes. 
Tabla 4. Indicadores de gestión de la carga turística de áreas protegidas

\begin{tabular}{|c|c|c|}
\hline Indicador & Norma & Método de evaluación \\
\hline $\begin{array}{l}\text { Distribución de los } \\
\text { visitantes en la } \\
\text { superficie asignada }\end{array}$ & $\begin{array}{l}\text { El margen de tolerancia es de } 1 \text { a } 2 \\
\text { [visitantes } / \mathrm{m}^{*} \text { ]. }\end{array}$ & $\begin{array}{l}\text { Durante el paso por puestos de } \\
\text { control de áreas protegidas. }\end{array}$ \\
\hline Erosión del sitio & $\begin{array}{l}\text { El límite máximo de degradación del } \\
\text { suelo es del } 10 \% \text {. }\end{array}$ & $\begin{array}{l}\text { A través de mapeo con base en } \\
\text { sistemas de información geográfica } \\
\text { (SIG) y análisis de suelos cada seis } \\
\text { meses. }\end{array}$ \\
\hline $\begin{array}{l}\text { Alteración del } \\
\text { ecosistema }\end{array}$ & $\begin{array}{l}\text { El margen de tolerancia es del } 10 \text { al } \\
20 \% \text { de pérdida de las especies } \\
\text { existentes. }\end{array}$ & Recuento de especies cada mes. \\
\hline $\begin{array}{l}\text { Afluencia de } \\
\text { visitantes }\end{array}$ & $\begin{array}{l}\text { El límite máximo de exceso de la } \\
\text { capacidad de carga es del } 15 \% \text {. }\end{array}$ & $\begin{array}{l}\text { Registro de ingreso de visitantes en } \\
\text { áreas protegidas. }\end{array}$ \\
\hline $\begin{array}{l}\text { Satisfacción de } \\
\text { visitantes }\end{array}$ & $\begin{array}{l}\text { El margen de tolerancia de } \\
\text { insatisfacciónde los visitantes es del } \\
20 \text { al } 30 \% \text {. }\end{array}$ & $\begin{array}{l}\text { Encuestas a visitantes y residentes de } \\
\text { sitios protegidos. }\end{array}$ \\
\hline $\begin{array}{l}\text { Daños a las } \\
\text { instalaciones }\end{array}$ & $\begin{array}{l}\text { El límite máximo de modificaciones } \\
\text { de la infraestructura es del } 20 \% \text {. }\end{array}$ & $\begin{array}{l}\text { Supervisión por parte de } \\
\text { guardaparques y pobladores de la } \\
\text { región. }\end{array}$ \\
\hline
\end{tabular}

Fuente: elaboración propia con base en Pedersen (2005).

Los indicadores de gestión de la carga turística de áreas protegidas permiten reflejar el estado de estos sitios, en lo que se refiere a su integridad y preservación de la biodiversidad. Si bien es importante vigilar el uso de las instalaciones y la satisfacción del visitante, también es indispensable vigilar de cerca la eficacia en la comunicación del significado histórico de estas ecorregiones.

\section{DISCUSIÓN DE RESULTADOS}

El dimensionamiento de la capacidad de carga turística física, real y efectiva dedicada a actividades agroecológicas se realizó con base en el método tradicional desarrollado para áreas protegidas (Cifuentes Arias et al., 1999). En este caso, se tomó en cuenta como factor limitante el daño a la flora y fauna, debido a que estos emplazamientos tienen fines de preservación que se extienden incluso a las labores agropecuarias llevadas a cabo en su interior.

La sucesión de las capacidades de carga calculadas y la cantidad registrada de visitantes (CRV) que realizan actividades agroecoturísticas en áreas protegidas nacionales se muestra en la Figura 5. 
Figura 5. Comparación de la secuencia de capacidades de carga

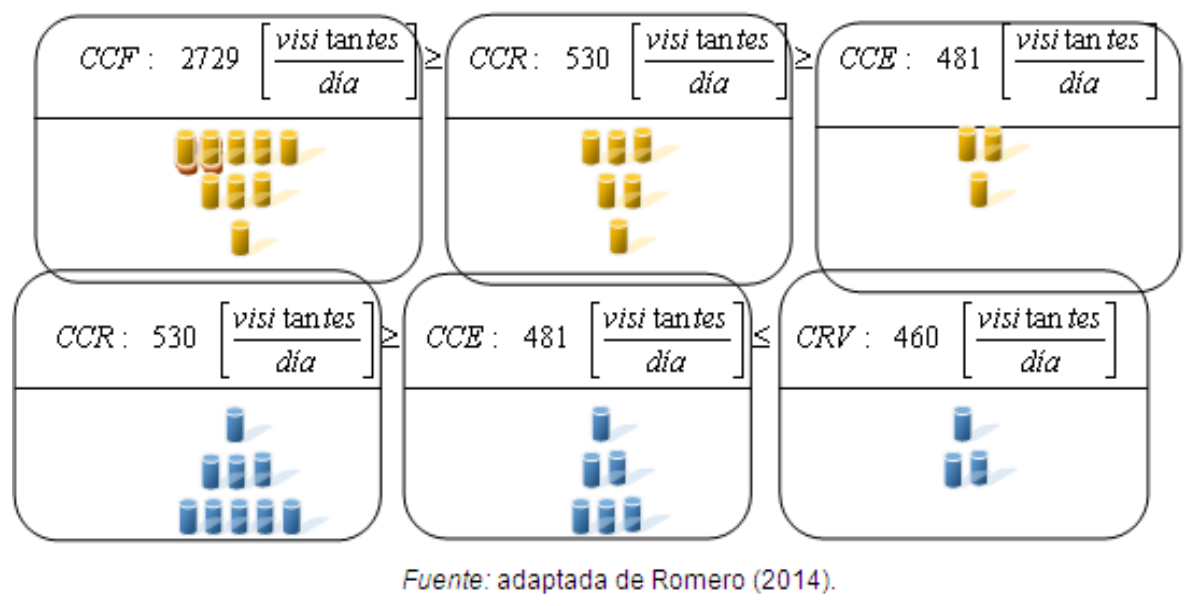

En base al análisis de esta información, es posible señalar que se tiene un margen de capacidad de carga de los sitios protegidos de 21 [visitantes/día]. Por tanto, no es necesario limitar el número de visitantes en estas zonas. Sin embargo, es posible realizar mejoras relativas a los factores de erodabilidad, accesibilidad y anegamiento que recibieron una notación poco favorable en este caso.

La Capacidad de Manejo (CM), es uno de los factores clave para la medición de la Capacidad de Carga Efectiva (CCE), puesto que refleja la eficiencia de gestión de las áreas protegidas para cumplir sus funciones y objetivos. El resultado de CM obtenido fue de 90,67\% en comparación a una situación óptima. Es importante destacar que la formación del personal, la infraestructura y el equipamiento no presentan deficiencias significativas. Cabe mencionar que la CM existente puede incrementarse en el marco de la mejora permanente de la gestión de sitios protegidos.

La valoración económica de la visita de sitios protegidos como bien ambiental, permite perfeccionar el método de dimensionamiento de la capacidad de carga turística de áreas protegidas, admitiendo estimar el valor monetario del beneficio ambiental y socioeconómico percibido por los turistas. Así como la generación de ingresos susceptibles de ser invertidos en la mejora de su funcionamiento y el desarrollo de las comunidades locales. En este caso, es posible evidenciar que la ganancia diaria de un área protegida individual corresponde a 404 Bs. en promedio. En algunos casos, este monto es insuficiente para cubrir los costos de funcionamiento de estos sitios, por lo que se justifica el hecho de que se requiera la cooperación internacional a fin de mantenerlos en operación.

\section{CONCLUSIONES}

La determinación de la capacidad de carga de actividades agroecoturísticas en áreas protegidas permite fijar límites respecto al número de personas que visitan estos sitios. Este indicador es susceptible de ser comparado con datos reales de afluencia de turistas a fin de verificar su pertinencia. El estudio permite comprobar que se opera al $96 \%$ de la capacidad de carga efectiva asignable al agroecoturismo en áreas protegidas nacionales. No obstante, esto puede evolucionar positivamente si se mejoran algunas de las características ecológicas, físicas y sociales de estos sitios; a fin de incrementar los ingresos económicos percibidos por la población local. 
Los impactos causados por el turismo deben ser identificados y supervisados con base en indicadores de gestión complementarios a la capacidad de carga de áreas protegidas. Aquellos pueden ser definidos en función a las expectativas de las partes interesadas, los objetivos organizacionales de dichos sitios, la situación ecológica, económica y social ideal.

Los datos de referencia reportados en la literatura son indispensables para el establecimiento de normas realistas de seguimiento de indicadores de gestión de carga turística en áreas protegidas. Se sugiere diseñar un inventario a través del que se pueda evidenciar la diferencia existente entre la situación actual y la óptima. Las normas deberán ajustarse a los objetivos de gestión de los sitios protegidos.

La economía ecológica es una herramienta complementaria al análisis de la capacidad de carga turística de áreas protegidas, debido a que es posible comparar la cantidad de visitantes máxima admisible en estos emplazamientos con los beneficios económicos generados de manera que se pueda evaluar la sostenibilidad social, ambiental y económica de las actividades agroecoturísticas llevadas a cabo en sitios protegidos.

El empleo de la economía ecológica permitirá realizar una mejor valorización y aprovechamiento de los recursos existentes en las áreas protegidas de interés nacional de Bolivia.

\section{REFERENCIAS}

- Amador, E., Cayot, L., Miguel, C., Cruz, E. y Cruz, F. (1996). Determinación de carga turística en los sitios de visita del Parque Nacional Galápagos. Ecuador: Instituto Ecuatoriano Forestal y de Áreas Naturales y Vida Silvestre.

- Aranguren, J., Moncada, J. A., Naveda, J., Rivas, D. y Lugo, C. (2008). Evaluación de la capacidad de carga turística en la playa de Conomita, municipio de Guanta, Estado Anzoátegui. Revista de Investigación, 64, 3161.

- Báez, A. L. y Acuña, A. (2003). Guía para mejores prácticas de ecoturismo en áreas protegidas. México: Comisión Nacional para el Desarrollo de Pueblos Indígenas.

- Botero Saltarén, C., Hurtado García, Y., González Porto, J., Ojeda Manjarrés, M. y Díaz Roca, L. H. (2008). Metodología de cálculo de la capacidad de carga turística como herramienta para la gestión ambiental. Gestión y Ambiente, 11(3), 109-122.

- Camacho Barreiro, A., \& Ariosa Roche, L. (2000). Dictionary of environmental terms. Cuba: Felix Varela Center.

- CESD. (2006). Una guía simple para la certificación del turismo sostenible y el ecoturismo. España.

- Cifuentes Arias, M., Mesquita, C. A., Méndez, J., Morales, M. E., Aguilar, N., Cancino, D. et al. (1999). Capacidad de carga turística de las áreas de uso público del monumento nacional Guayabo, Costa Rica. WWF Centroamericana, 58.

- Cifuentes, M. (1992). Determinación de capacidad de carga turística en áreas protegidas. Turrialba, Costa Rica: Centro Agronómico Tropical de Investigación y Enseñanza -CATIE-.

- Coccossis, H., Mexa, A., Collovini, A., Parpairis, A. y Konstandoglou, M. (2001). Defining, meausuring and evaluating carrying capacity in european tourism destinations. Athens, Greece: University of the Aegean. 
- El-Hage Scialabba, N. y Williamson, D. (2004). The scope of organic agriculture, sustainable forest management and ecoforestry in protected area management. Environmental and Natural Resources, 18, 1-49.

- Martínez Alier, J., Roca, J. y Sánchez, J. (1998). Curso de economía ecológica. Programa de las Naciones Unidas para el Medio Ambiente (PNUMA)-Oficina Regional para América Latina y El Caribe. México: Serie de Textos Básicos para la Formación Ambiental, 1, 1-132.

- Pedersen, A. (2005). Gestión del turismo en sitios del Patrimonio Mundial. Francia: Organización de las Naciones Unidas para la Educación, la Ciencia y la Cultura -UNESCO-.

- Puente Santos, E. D., Pérez Ramírez, C. A. y Solís Barrón, C. I. (2011). Capacidad de carga en senderos turísticos del centro de cultura para la conservación "Piedra Herrada" de México. Quivera, 13(2), 93-114.

- Romero García, O. (2014). Capacidad de carga turística de la reserva ecológica mineral de Nuestra Señora de Cosalá, Sinaloa, México. AgroProductividad, 30-34.

- SERNAP -Servicio Nacional de Áreas Protegidas-. (2007). Informe sobre el Sistema Nacional de Áreas Protegidas de Bolivia. II Congreso Latinoamericano de Parques Nacionales y Otras Áreas Protegidas. Bolivia.

- SERNAP -Servicio Nacional de Áreas Protegidas-. (2011). Estrategia para el Desarrollo del Turismo en el Sistema Nacional de Áreas Protegidas. Estado Plurinacional de Bolivia: Ministerio de Medio Ambiente y Agua.

- Tudela Serrano, M. L. y Giménez Alarte, A. I. (2008). Determinación de la capacidad de carga turística en tres senderos de pequeño recorrido en el municipio de Cehegín (Murcia). Cuaderno de Turismo, 22, 211-229.

- Verner, D., Andersen, L., Brisson, I., Hesselbjerg Christensen, J., Bjerg Geary, J., Kronik, J. y Tikjob, S. A. (2010). Reducing poverty, protecting livelihoods and building assets in a changing climate. Social implications of climate change for Latin America and the Caribbean. Washington D.C., USA: The World Bank.

- Von Lossau, A. y Li, Q. (Eds.). (2011). Sourcebook on Sustainable Agrobiodiversity Management. República Popular China: Social Sciences Academy Press.

1. Universidad Europea en Energía y Medio Ambiente (UEMA), Doctor en Sostenibilidad. Programa Doctoral en Sostenibilidad, España. Valladolid, España. marianameaveacua@yahoo.es

2. Universidad Europea en Energía y Medio Ambiente (UEMA), Doctor en Estrategias para el Desarrollo Agrícola Regional. Programa Doctoral en Sostenibilidad, España. Valladolid, España. morin@colpos.mx

Para citar este artículo: Meave Acuña, M. y Lugo-Morin, D. R. (2016). Capacidad de carga asignable al agroecoturismo en áreas protegidas de Bolivia. Revista Luna Azul, 42, 89-104. Recuperado de http://200.21.104.25/lunazul/index.php?option=com_content\&view=article\&id=130 